\title{
Reaching training using sticky mittens as an early intervention protocol for an infant under environmental risk for motor delay: single-case research
}

\section{CAMILA A. S. SANTANA ${ }^{1}$ | ANA LUIZA R. GRECO ${ }^{1}$ | CAROLINA F. DA SILVA ${ }^{1}$ | ELOISA TUDELLA ${ }^{1}$}

1Department of Physical Therapy of the Federal University of São Carlos. Federal University of São Carlos- UFSCar-Brazil. Washington Luís Highway, km 235 - SP-310, Post Code 13565-905. São Carlos City, São Paulo State - Brazil.

Correspondence to:Ana Luiza Righetto Greco. Department of Physical Therapy of the Federal University of São Carlos. Federal University of São Carlos- UFSCar-Brazil. Washington Luís Highway, km 235 - SP-310, Post Code 13565-905. São Carlos City, São Paulo State - Brazil.

email: analuiza.nenem@gmail.com

https://doi.org/10.20338/bjmb.v14i2.172

\section{HIGHLIGHTS}

- Low socioeconomic status is an important risk factor for neurosensorimotor delays in infants.

- Affordable early intervention strategies are needed for infants from low-income families exposed to the risk of neurosensorimotor delay.

- The use of sticky mittens during reaching training could be a suitable strategy, as this has been shown to improve aspects of reaching ability in a 3 month old infant of lowincome.

- Improvement in one specific motor ability could not easily be transferred into more complex motor abilities.

$\begin{array}{ll}\text { ABBREVIATIONS } \\ \text { AIMS } & \text { Alberta Infant Motor Scale } \\ \text { AHEMD-IS } & \text { Home Environment for Motor } \\ & \text { Development-Infant Scale } \\ \text { F1 } & \text { follow-up 1 } \\ \text { F2 } & \text { follow-up 2 } \\ \text { high-SES } & \text { High socioeconomic status } \\ \text { ICF } & \text { International Classification of } \\ & \text { Functioning, Disability and Health } \\ \text { low-SES } & \text { Low socioeconomic status } \\ \text { PIR } & \text { Poverty Income Ratio } \\ \text { PT } & \text { pre-training } \\ \text { PT1 } & \text { post-training 1 } \\ \text { PT2 } & \text { post-training 2 } \\ \text { RT } & \text { retention } \\ \text { SES } & \text { socio-economic status }\end{array}$

BACKGROUND: Low socioeconomic status (low-SES) is an important environmental risk factor for developmental delay in infants. Access to affordable early intervention strategies may be limited for this population. As it is a low cost and easily reproducible intervention, reaching training using sticky mittens as a facilitator can be easily conducted by parents at home or in clinics, improving manual ability and exploration, and thus helping to avoid possible motor delays in infants from low-SES families.

AIM: To investigate the effects of reaching training using sticky mittens on manual reach and gross motor development of a 3 month-old full-term infant with low-SES.

METHOD: The training using sticky mittens occurred on 5 consecutive days, for 20 minutes per day. A total of 6 assessments were performed to measure: the number of reaches, proximal and distal adjustments (Kinovea), gross motor development (AIMS), and quality/quantity of affordances at home (AHEMD-IS).

RESULTS: After the training protocol, improved reaching ability was observed, with an increase in the number of reaches and bimanual adjustments, with hands open and verticalized. No improvements in other gross motor skills were observed.

CONCLUSION: Reaching training using sticky mittens seems to be a potential and affordable early intervention strategy for infants from low-SES families at risk for motor delay.

KEYWORDS: Protocol | Infant | Early intervention | Reaching | Environmental factors

\section{INTRODUCTION}

Based on multiple views about human development, the framework of the International Classification of Functioning, Disability and Health (ICF) 1 proposed by the World Health Organization comprehends a biopsychosocial perspective that views health as the result of the interaction of the health condition with Contextual Factors (Environmental and/or Personal Factors), Body Functions and Structures, and Activity and 
Participation. This framework is helpful to expand the view about the multiple biological and environmental factors and general determinants related to the development of young children. ${ }^{2}$

The environmental factors encompass the attitudinal, physical, and social environment in which people live and conduct their lives. Hence, the child's development is a result of the interaction of all these factors, where each can act as a barrier or a facilitator to development. ${ }^{3}$ For this reason, developmental delays in infancy can be caused by exposure to several risk factors related to environmental aspects, such as low parental education, lack of family structure, poor home opportunities for development, and poverty. ${ }^{4}$ An individual is in a poverty situation when there is no adequate access to basic needs such as housing, medical care, and public services. ${ }^{5}$ Poverty situations are frequently associated with parental lack of income to invest in educational toys, which could contribute to adequate child development. ${ }^{2}$ Usually, infants from families with low socioeconomic status (low-SES) do not receive the necessary health support unless they present a particular health condition, such as the presence of genetic syndromes, congenital malformations, or premature birth. ${ }^{6}$

The consequences of poverty in the early stages of child development may be a barrier to their development in later stages. ${ }^{7}$ There is evidence of the negative impact of being exposed to low-SES on visual attention, ${ }^{8}$ cognition, ${ }^{9}$ and strategies of manual exploration in infants between 6 and 12 months of age when compared with high socioeconomic status infants ${ }^{6}$ (high-SES). Infants living in unfavorable environmental conditions may therefore present developmental delays. ${ }^{6}$

Research shows that children from low-income families have twice the chance of motor delays when compared to children from families with better economic conditions. The period of life, duration, and context in which the child is exposed to poverty should also be considered.10 Thus, as multiple environmental factors can be associated with motor and other developmental outcomes in young children, health professionals must identify infants exposed to risk situations as early as possible, such as those living in poverty or in low-income families, in order to plan age-appropriate motor and cognitive interventions aiming to avoid future delays. In this context, Heathcock et al. (2008)11 found that incorporating early motor interventions into infant and family lives can eliminate the delay in reaching for objects in preterm babies at risk for motor delays. Therefore, reaching and grasping training are examples of motor abilities that can be trained early in life, and that may influence the overall development of the infant throughout the first year of life. ${ }^{11}$

Learning to reach for, grasp, and explore objects with their hands allows infants to learn about object characteristics and, consequently, about how these can be used as tools to achieve goals and problem-solve. ${ }^{12}$ These new skills can increase the motivation to explore their surroundings, making infants express a young human competence to understand the physical objects of the environment, and motivate them to explore new intriguing events, exercising their new motor abilities and feeling rewarded by practicing them. ${ }^{12}$

Thus, based on the roles of the environment on motor development, providing early opportunities for infants to develop and practice their emerging motor skills could be an excellent strategy to avoid developmental delays in infants exposed to environmental risks. When planning intervention strategies involving low-SES infants and families, the choice of affordable, common, or reusable materials could be an important factor allowing the families to conduct these strategies at home as an extension of the clinic-based 
therapy time. Based on this, reach training using sticky mittens could be a good strategy to improve new learning opportunities, boosting infant object exploration and acquisition of future skills in multiple domains. ${ }^{13}$

There is evidence of positive effects of providing early active manual experiences using sticky mittens in premature ${ }^{14}$ and full-term infants ${ }^{15}$, and in infants at risk for autism. ${ }^{16}$ It was shown that this type of approach can sustain the motivation of the infant during the exploration, allowing the transfer of a new ability to the execution of other abilities over time, a process that could be slower without sticky mittens. ${ }^{17}$ An increase in the total number of reaches was described by Wiesen, Watkins, and Needham (2016)12, who verified that not only the number of reaches improved after the training, but also the grasping ability. Additionally, when the reaching training using sticky mittens was performed at home by parents, positive effects were observed not only in the behavior of the reach, grasp, and exploration of the object but also in the social behavior of infants during play. ${ }^{16}$

As low-SES families may face challenges to access affordable early interventions, low-cost and easily replicable strategies that can be conducted by parents at home, such as reaching training using sticky mittens, could be an option aimed at avoiding possible motor delays. However, this strategy has not yet been tested in this population. Thus, the current pilot single-case research aimed to explore the effects of a reaching training protocol using sticky mittens on the manual reaching performance of an infant exposed to environmental risk for motor delay due to low-SES and investigate if there is any repercussion of the training on gross motor development. We hypothesized that the reaching training protocol using sticky mittens would improve the reaching ability of this infant, and may facilitate the acquisition of other gross motor abilities. The results of this research could provide clinicians with an option to develop and adapt early intervention strategies for infants from low-SES families.

\section{METHODS}

\section{Participant characterization}

One typical full-term male infant took part in this study, 3 months and 16 days of age, and weighing $6.945 \mathrm{~kg}$ on the day of the first assessment. He received an Apgar score of 9 in the first minute and 10 in the fifth minute after birth, without any associated conditions or cognitive concerns. In the first assessment, the infant was at the phase of acquisition of the manual reaching ability, performing between three and five reaches in a minute, and presenting no motor delay for his age according to the AIMS scale. The participant was chosen by convenience based on a possible patient list from the main public maternity hospital in the city of São Carlos-SP, Brazil.

The socio-economic status (SES) of the infant was determined according to the Poverty Income Ratio (PIR) in combination with the maternal level of education. This measure gives us three possible socio-economic parameters: a) Low SES (incomplete high school and PIR less than 2); b) Middle SES (complete high school and independent PIR, incomplete high school and PIR greater than or equal to 2, complete high school and PIR less than or equal to 2); or c) High SES (higher education and PIR greater than or equal to 2). Based on these parameters, the infant in this study was classified as low SES, with a PIR of 0.7 . 
In addition, according to household income, consumer goods, use of public services, and education of the person who made the largest income contribution in the house, the economic status of the infant's family using the "Brazilian Economic Classification Criterion of Brazilian Association of Research Companies" tool was estimated. According to this measure, the infant had a score of 15 points, corresponding to a family average monthly income of 198.36 USD.

The research was approved by the ethical committee of the Federal University of São Carlos ( $n^{0}$ 2096695). The parents signed and received a copy of the consent term to participate in this study.

\section{Assessments and instruments}

A total of 6 assessments were performed, as follows: 1) pre-training (PT): manual reaching assessed using Kinovea software (performed specifically between 2-4 days after identification of manual reaching onset at the infant home), gross motor development assessed using the Alberta Infant Motor Scale (AIMS), and quality and quantity of affordances in the home environment assessed using the Home Environment for Motor Development-Infant Scale (AHEMD-IS); 2) post-training 1 (PT1): manual reaching, performed immediately after the first training session; 3) post-training 2 (PT2): manual reaching, performed immediately after the end of the complete training protocol; 4) retention (RT): manual reaching, performed within 24 hours after the end of the training protocol; 5) follow-up 1 (F1): gross motor development, performed 1 month after the end of the training protocol; 6) follow-up 2 (F2): gross motor development, performed 2 months after the end of the training protocol. Each assessment had an average duration of 2 minutes.

To assess the quality and quantity of affordances in the home environment the AHEMD-IS was used. This is a parental self-reporting instrument for children between 3 and 11 months of age, composed of four dimensions: stimulation diversity; fine motor skill toys; gross motor skill toys; and physical space of the residence (external and internal). According to the total score obtained from all dimensions, the environment can be categorized as: a) less than adequate, b) moderately adequate, c) adequate, and d) excellent.

The manual behavior was video recorded and analyzed through the software Kinovea 0.8.21. For this, passive reflexive markers were affixed on the infant's wrists, enabling the software to identify the position of the upper limbs in the three-dimensional space. Reaches were quantified by the total number of reaches performed in each evaluation. The proximal adjustments considered were unimanual or bimanual reaching, and the distal adjustments considered were hand orientation and hand opened/closed. The proximal and distal adjustments were quantified through the percentage of frequency of adjustments made in each evaluation.

Gross motor development was assessed through the observational instrument AIMS and compared with the scale normative data. For the total score, the score of each subscale (prone, supine, sitting, and standing) is added, and according to the scale normative data chart for each age, the percentile of motor performance is obtained. The gross motor development may be categorized as: late (percentile $\leq 10)$; at risk for delay (percentile between $\geq 11$ and $<25$ ); and normal $(\geq 25)$.

\section{Training protocol}


The mittens were made with cotton sock fabric with Velcro® strips stuck on the dorsal and ventral regions. The mittens involve only the region of the infant's metacarpals, allowing the phalanges to move freely and have greater contact with the training toys (figure 1). Velcro® strips were also added to the training toys, enabling these to adhere to the mittens (figure 2). The toys used were soft and rubbery, unfamiliar, and attractive to the infant. The Velcro® component in the mittens and on the toys is one of the key components of the protocol, enabling the infant to easily and independently explore the toys.

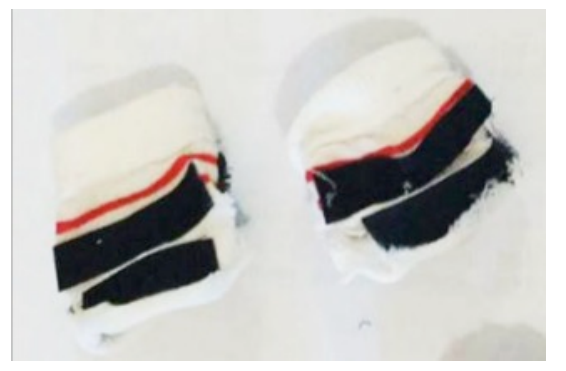

Figure 1. Model of sticky mittens used during the training protocol.

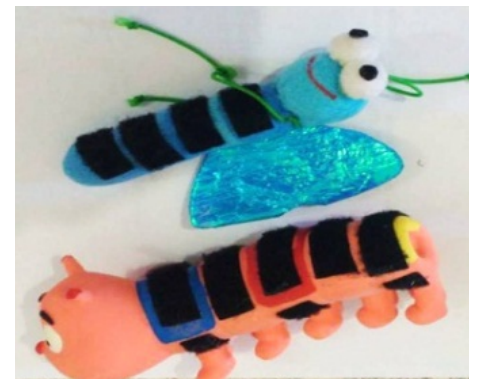

Figure 2. Example of toys used during the training protocol.

The protocol was applied for 20 minutes per day, on 5 consecutive days, making 100 minutes of training in total. The training protocol consisted of three different activeassisted activities based on the studies of Needham, Barrett, and Peterman (2002) ${ }^{18}$, and Williams, Corbetta, and Guan (2015) ${ }^{19}$. Thus, to conduct the training, immediately after the PT assessment, the infant was positioned reclined on the therapist's thighs, sitting with the trunk supported on the therapist's legs, who was sitting on the floor. A small pillow was placed on the therapist's knees to support the infant's head, favoring a face-to-face interaction while the neck of the infant remained in semi-flexion, which also facilitates the alignment between head and trunk and the hands at the midline, in the infant's visual field. The training protocol was also explained to the parents, who were trained by the therapist to perform the protocol daily at home during the study period.

All the evaluations and training protocol took place at the Research Laboratory in Motion Analysis (LAPAM) at the Federal University of São Carlos, and were conducted by a physiotherapist with experience in early intervention, and trained to perform this specific protocol. During all evaluations and training, the mother of the infant was allowed to breastfeed or calm the infant if necessary.

The percentage change across assessments for each variable of interest was calculated. The data are shown in a descriptive format.

\section{RESULTS}

\section{Home environment}

According to the results of the AHEMD-IS at the PT assessment, the overall quantity and quality of the motor stimulation opportunities present in the home environment were classified as less than adequate (total score: 13). The specific dimension scores were: variety of stimulation: moderately adequate (Score: 10); fine-motor toys: less than 
adequate (Score: 1); gross-motor toys: less than adequate (Score: 1); physical space of the residence (external and internal): less than adequate (Score: 1).

\section{Manual reaching}

Reaching performance improved after the training protocol. Regarding the number of reaches, there was a decrease of $60 \%$ from PT to PT1. At PT2, the infant showed an increase of $50 \%$ in the number of reaches when compared with PT. At RT, a decrease of $30 \%$ was observed in the number of reaches when compared to PT2. However, it is worth mentioning that the number of reaches at RT was still $20 \%$ higher than PT (Figure 3).

Regarding proximal adjustments, there was a decrease in unimanual adjustments from PT $(90 \%)$ to PT1 (66.6 \%). Immediately after the application of the training, there was an increase in the number of bimanual reaches at PT2 $(60 \%)$ when compared to PT (10\%). However, 24 hours after the training, there was a decrease in bi-manual reaching from PT2 $(60 \%)$ to RT $(57.1 \%)$. It is important to emphasize that from PT to RT, the lowSES infant performed more bi-manual reaches (10\% versus $57.1 \%$, respectively) (Figure 4).

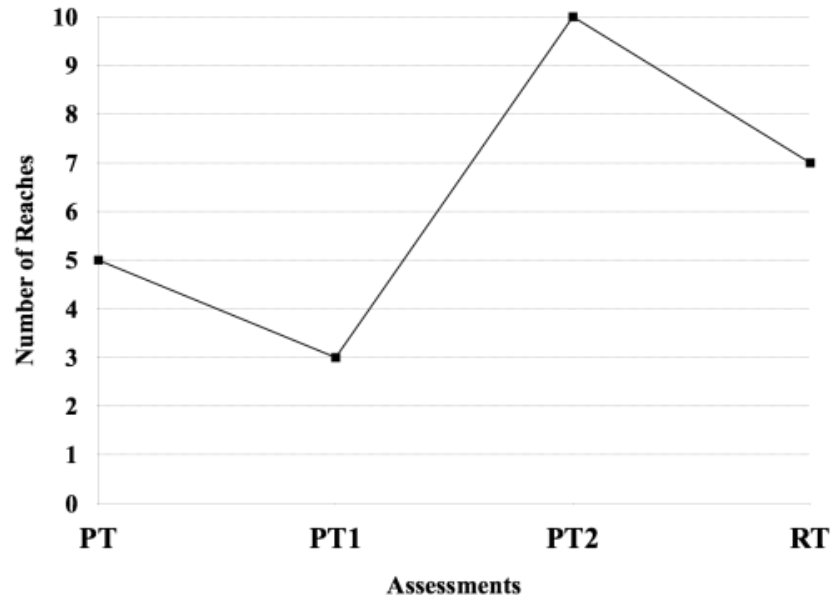

Figure 3. Numbers of reaches. PT: pre-training; PT1: post-training 1; PT2: post-training 2; RT: retention.

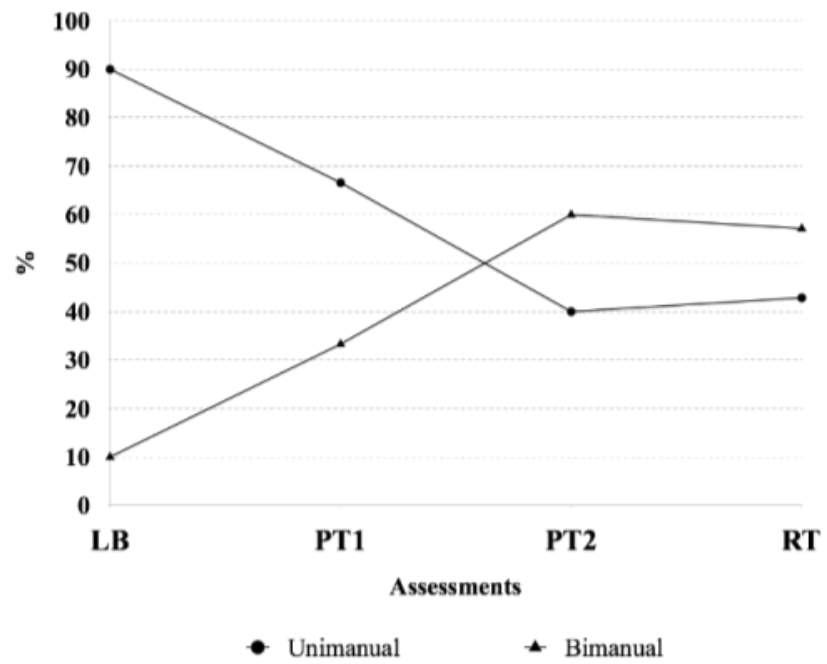

Figure 4. Proximal Adjustments. PT: pre-training; PT1: post-training 1; PT2: post-training 2; RT: retention. 
Regarding distal adjustments, an increase in open hand reaches was observed from PT $(90 \%)$ to PT1 (100\%). After the application of 5 training sessions, there was greater variability in distal adjustment strategies, with the infant performing $50 \%$ of the adjustments with an open hand and $50 \%$ with a semi-open hand at PT2 when compared to PT. At 24 hours after the application of the training, an increase in the open hand reaching was verified at PT2 (50\%) compared to RT (70 \%) (Figure 5).

An increase in reaches with a verticalized hand was observed immediately after one training session (PT: $0 \%$ versus PT1: $25 \%$ ). After the practice of the whole training protocol, there was a greater increase in the movements with a vertical hand (PT: $0 \%$ versus PT2: $40 \%$ ). At 24 hours after the end of the training, the infant continued to perform reaches with vertical hand orientation (PT2: $40 \%$ versus RT: $100 \%$ ). It is worth mentioning that the reaching behavior pattern improved after the protocol, as seen by the increased percentage of reaches performed with a vertical hand at RT (100\%) compared to PT (0\%) (Figure 6).

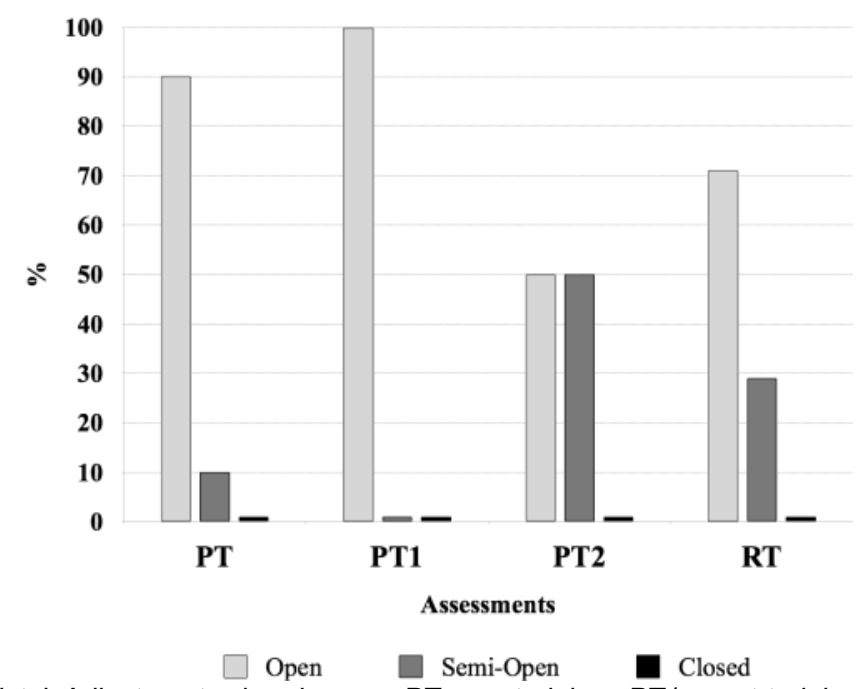

Figure 5.Distal Adjustments: hands open.PT: pre-training; PT1: post-training 1;PT2: post-training 2; RT: retention.

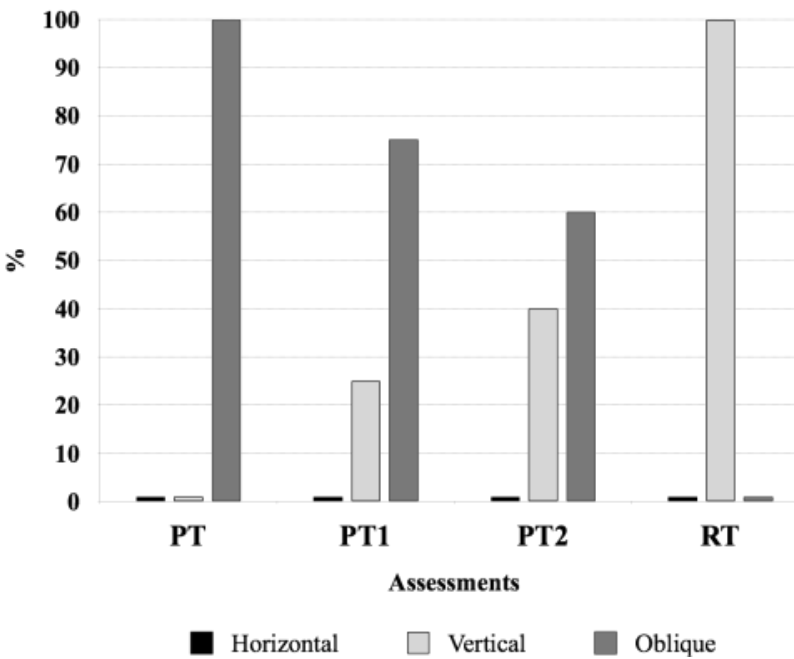

Figure 6.Distal Adjustments: hand orientation.PT: pre-training; PT1: post-training 1;PT2: post-training 2; RT: retention. 


\section{Gross motor development}

The gross motor development of the infant was compared with the AlMS normative data. An increase was observed in the number of motor skills from PT to F1 and F2. However, the final percentile score for his age showed a risk for motor delay at the PT and F1 assessments, and a decline to late at the F2 assessment (Table 1).

\begin{tabular}{cccc} 
Table 1 - AIMS scale score. & PT & F1 & F2 \\
\hline AIMS subscale & 3 & 4 & 6 \\
\hline Prone & 3 & 5 & 4 \\
Supine & 2 & 2 & 2 \\
Sitting & 1 & 2 & 2 \\
Standing & $\mathbf{9}$ & $\mathbf{1 3}$ & $\mathbf{1 4}$ \\
$\begin{array}{c}\text { Total Score } \\
\text { \% rank score }\end{array}$ & 14 & 12 & 5 \\
$\begin{array}{c}\text { according to age } \\
\text { Final gross motor }\end{array}$ & at risk for delay & at risk for delay & late \\
$\begin{array}{c}\text { function categorization } \\
\text { PT: pre-training; F1: follow-up 1; F2: follow-up 2. }\end{array}$ & &
\end{tabular}

\section{DISCUSSION}

This study aimed to explore the effects of a reaching training protocol using sticky mittens on manual reaching performance of an infant exposed to environmental risk due to low-SES and analyze if there was any further repercussion of the training on gross motor functions. Based on the results, our hypothesis that the reaching training using sticky mittens could improve reaching ability was confirmed since the infant showed better reaching performance after the training. However, our second hypothesis that the improvements in manual reach could facilitate the acquisition of other gross motor functions was not confirmed since no additional improvements in other motor abilities were observed over time.

Regarding the immediate results of the reaching training with sticky mittens, Nascimento et al. (2019) 20 found changes in the exploration of objects after a single active training session of only 10 minutes. For the infant evaluated in our study, it was not possible to verify changes in the number of reaches after only a single session of 20 minutes even with sticky mittens as a facilitator. This may be due to contextual individualities of only a single participant or to the fact that in the study of Nascimento et al. $(2019)^{20}$, the training was performed only actively by the infants, and in our protocol, the training occurred in an active and active-assisted format.

In the matter of the proximal adjustments, it was observed that the infant presented more unimanual reaching at PT and began to perform more bimanual reaches immediately after one session and also after the entire training protocol. We believe that the variety, malleability, and medium size of the toys used in the training facilitated the exploration and selection of the best performance strategy, which resulted in the infant being able to perform the adjustments with two hands. The same effect was observed in 
the study of Wiesen, Watkins, and Needham (2016) $)^{12}$ and Nascimento et al. (2019) ${ }^{20}$, in which the bimanual strategy was also adopted. In contrast to using one hand, the use of both hands to explore the object may provide the infant with more exploration options. ${ }^{21}$

Concerning the distal adjustments, immediate effects were observed after a single session and after the whole training protocol for the hand orientation. De Toledo, Soares, and Tudella (2011)22 observed a predominance of oblique hand orientation when infants were sitting in a reclined position. This was also observed in our PT assessment. However, the infant progressed from oblique to vertical hand orientation after a single 20 minute training session, which remained after completing the 100 minutes of training. We believe that the movement components of the active-assisted activities during the training, which includes the supination of the infant's hands, may have facilitated the adoption of vertical hand orientation soon after a single session, providing better positioning of the hand on the object during grasping.

In the study by Heathcock, Lobo, and Galloway (2008)11, training without sticky mittens did not result in changes in distal adjustments in preterm infants, and the number of reaches with open hands only changed after 8 weeks of daily training. However, in our study, the infant performed reaches with an open hand at PT and PT1, and the number of reaches with the semi-open hand increased at PT2. This same behavior was observed by Cunha, Woollacott, and Tudella, (2013)21, where after the training the reaching behavior was consistent with the semi-open hand, and the authors attributed this change to the fact that similarly to the hand orientation, the opening of the hand occurs from the infant's perception of the properties of the object. Likewise, we believe that at PT1, the infant performed all reaches with an open hand as a result of the training characteristics, thus choosing this as the best strategy to explore the object. However, during the following assessments, we believe that the infant was going through an intense exploration phase to choose the best strategy that could be the most adequate to reach the object in a more precise and efficient way.

Success in performing basic tasks during childhood can promote competencies for subsequent developmental abilities. ${ }^{23}$ An infants' own experiences and interactions with their bodies, such as those experienced during the training activities using sticky mittens, could have beneficial effects on learning and development processes ${ }^{24}$. Based on this, we expected that improvement in an important motor skill such as manual reaching could facilitate the infant's exploration and interaction with the environment. However no repercussions of the hand experiences on the infant's subsequent gross motor development were observed according to the results of the AIMS scale, which showed a risk of motor delay at PT and P1, and motor delay two months after the end of the training.

It seems that with development, as the abilities become more complex, the infant starts to face more challenges to overcome the new development demands. It is important to note that the infant's home was classified as having less than adequate opportunities for good development according to the AHEMD-IS, a fact that may be directly related to the poor gross motor development of the infant, since the presence of stimuli and an adequate environment have major influences on development.

According to Adolph et al. (2012)25, low-SES, physical environment, and affordances present at home could frequently be barriers to exploration, and the low amount of time that infants spend moving through the environment contributes to the poor performance observed in this population. Additionally, interventions performed in laboratories or clinics may not present the same results as interventions performed at 
home as they are not being conducted in the same environment where the infant's daily routine occurs. Research shows that when parents can incorporate early motor stimulation into the family routine the results are more positive. ${ }^{24}$

Thus, it is important to highlight the need for early interventions in developing countries due to the high incidence of infants exposed to the risk of motor delay as a consequence of low-SES. Considering the simplicity and accessibility of reaching training using sticky mittens, ${ }^{12}$ this could be an easily applicable option for families of low-SES. Thus, based on the data obtained from this single-case research, we can highlight the importance of providing early stimulation at clinics and also of the promotion of stimulation by parents at home, which, combined, could contribute to the development of infants exposed to environmental risk factors.

\section{Study limitations}

As this is a single-case study, we strongly suggest the development of further studies with a larger population, making it possible to better understand the training effects and expand the findings to the general population of infants with low-SES. Additionally, we encourage researchers to conduct randomized clinical trials exploring the protocol in comparison with other populations and also other intervention strategies for infants at risk for motor delay.

\section{CONCLUSION}

According to our results, the reaching training protocol using sticky mittens was effective to improve the reaching performance of the assessed infant, increasing bimanual reaches, with hands open and with vertical orientation. No transference of the improvements in manual ability to gross motor performance was found.

\section{REFERENCES}

1. World Health Organization. International Classification of Functioning, Disability and Health (ICF). In:; 2001. doi: https://www.who.int/classifications/icf/en/.

2. Hwang A-W, Liao H-F, Chen P-C et al. Applying the ICF-CY framework to examine biological and environmental factors in early childhood development. J Formos Med Assoc. 2014;113(5):303-312. doi:10.1016/j.jfma.2011.10.004.

3. Bradley RH, Burchinal MR, Casey PH. Early Intervention: The Moderating Role of the Home Environment. Appl Dev Sci. 2001;5(1):2-8. doi:10.1207/S1532480XADS0501_1.

4. Pereira KR, Valentini NC, Saccani R. Brazilian infant motor and cognitive development: Longitudinal influence of risk factors. Pediatr Int. 2016;58(12):1297-1306. doi:10.1111/ped.13021.

5. Short KS. Child Poverty: Definition and Measurement. Acad Pediatr. 2016;16(3):S46-S51. doi:10.1016/j.acap.2015.11.005.

6. Clearfield MW, Bailey LS, Jenne HK, Stanger SB, Tacke N. SOCIOECONOMIC STATUS AFFECTS ORAL AND MANUAL EXPLORATION ACROSS THE FIRST YEAR. Infant Ment Health J. 2014;35(1):63-69. doi:10.1002/imhj.21423. 
7. Yoshikawa H, Aber JL, Beardslee WR. The effects of poverty on the mental, emotional, and behavioral health of children and youth: Implications for prevention.Am Psychol. 2012;67(4):272-284. doi:10.1037/a0028015.

8. Clearfield MW, Jedd KE. The Effects of Socio-Economic Status on Infant Attention. Infant Child Dev. 2013;22(1):53-67. doi:10.1002/icd.1770.

9. Clearfield MW, Niman LC. SES affects infant cognitive flexibility. Infant Behav Dev. 2012;35(1):29-35. doi:10.1016/j.infbeh.2011.09.007.

10. Chaudry A, Wimer C. Poverty is Not Just an Indicator: The Relationship Between Income, Poverty, and Child Well-Being. Acad Pediatr. 2016;16(3):S23-S29. doi:10.1016/j.acap.2015.12.010.

11. Heathcock JC, Lobo M, Galloway JC (Cole). Movement Training Advances the Emergence of Reaching in Infants Born at Less Than 33 Weeks of Gestational Age: A Randomized Clinical Trial. Phys Ther. 2008;88(3):310-322. doi:10.2522/ptj.20070145.

12. Wiesen SE, Watkins RM, Needham AW. Active Motor Training Has Long-term Effects on Infants' Object Exploration. Front Psychol. 2016;7. doi:10.3389/fpsyg.2016.00599.

13. Bornstein MH, Hahn C-S, Suwalsky JTD. Physically Developed and Exploratory Young Infants Contribute to Their Own Long-Term Academic Achievement. Psychol Sci. 2013;24(10):1906-1917. doi:10.1177/0956797613479974.

14. Karlamangla AS, Merkin SS, Crimmins EM, Seeman TE. Socioeconomic and Ethnic Disparities in Cardiovascular Risk In the United States, 2001-2006.Ann Epidemiol. 2010;20(8):617-628. doi:10.1016/j.annepidem.2010.05.003.

15. Cunha AB, Lobo MA, Kokkoni E, Galloway JC, Tudella E. Effect of Short-Term Training on Reaching Behavior in Infants: A Randomized Controlled Clinical Trial. J Mot Behav. 2016;48(2):132-142. doi:10.1080/00222895.2015.1050549.

16. Libertus K, Landa RJ. Scaffolded reaching experiences encourage grasping activity in infants at high risk for autism.Front Psychol. 2014;5. doi:10.3389/fpsyg.2014.01071.

17. Needham AW, Wiesen SE, Hejazi JN, Libertus K, Christopher C. Characteristics of brief sticky mittens training that lead to increases in object exploration. J Exp Child Psychol. 2017;164:209-224. doi:10.1016/j.jecp.2017.04.009.

18. Needham A, Barrett T, Peterman K. A pick-me-up for infants' exploratory skills: Early simulated experiences reaching for objects using 'sticky mittens' enhances young infants' object exploration skills. Infant Behav Dev. 2002;25(3):279-295. doi:10.1016/S01636383(02)00097-8.

19. Williams JL, Corbetta D, Guan Y. Learning to reach with "sticky" or "non-sticky" mittens: A tale of developmental trajectories. Infant Behav Dev. 2015;38:82-96. doi:10.1016/j.infbeh.2015.01.001.

20. Nascimento AL, Toledo AM, Merey LF, Tudella E, Soares-Marangoni DA. Brief reaching training with "sticky mittens" in preterm infants: Randomized controlled trial. Hum Mov Sci. 2019;63:138-147. doi:10.1016/j.humov.2018.11.015. 
Brazilian Journal of Motor Behavior

21. Cunha AB, Woollacott $M$, Tudella $E$. Influence of specific training on spatio-temporal parameters at the onset of goal-directed reaching in infants: a controlled trial. Brazilian $\mathrm{J}$ Phys Ther. 2013;17(4):409-417. doi:10.1590/S1413-35552013005000099.

22. de Toledo AM, de Almeida Soares D, Tudella E. Proximal and Distal Adjustments of Reaching Behavior in Preterm Infants. J Mot Behav. 2011;43(2):137-145. doi:10.1080/00222895.2011.552076.

23. Engle WA, Tomashek KM, Wallman C. "Late-Preterm" Infants: A Population at Risk. Pediatrics. 2007;120(6):1390-1401. doi:10.1542/peds.2007-2952.

24. Mahoney G, Perales F. The role of parents in early motor intervention. Downs Syndr Res Pract. 2006;10(2):67-73. doi:10.3104/reviews.307.

25. Adolph KE, Cole WG, Komati M, et al. How Do You Learn to Walk? Thousands of Steps and Dozens of Falls per Day. Psychol Sci. 2012;23(11):1387-1394. doi:10.1177/0956797612446346.

\section{ACKNOWLEDGMENTS}

We would like to acknowledge all our colleagues at the Núcleo de Estudos em Neuropediatria e Motricidade (NENEM-UFSCar) involved in this Project.

Citation: Santana CAS, Greco ALR, da Silva CF, Tudella E.Reaching training using sticky mittens as an early intervention protocol for an infant under environmental risk for motor delay: single-case research. BJMB. 2020: 14(2): 85-96.

Editors: Dr Fabio Augusto Barbieri - São Paulo State University (UNESP), Bauru, SP, Brazil; Dr José Angelo Barela São Paulo State University (UNESP), Rio Claro, SP, Brazil; Dr Natalia Madalena Rinaldi - Federal University of Espírito Santo (UFES), Vitória, ES, Brazil.

Copyright:@ 2020 Santana, Greco, da Silva and Tudella and BJMB. This is an open-access article distributed under the terms of the Creative Commons Attribution-NonCommercial-NoDerivatives 4.0 International License which permits unrestricted use, distribution, and reproduction in any medium, provided the original author and source are credited. Funding: This work has no funding support.

Competing interests: The authors have declared that no competing interests exist.

DOl:https://doi.org/10.20338/bjmb.v14i2.172 